\title{
Prognostic Role of Cardiac Magnetic Resonance Imaging in Patients with Previous Myocardial Infarction: A Clinical Dataset
}

\author{
Gianluca Di Bella ${ }^{a}(\bowtie), G i o v a n n i$ Aquaro ${ }^{b}$, Francesca Mastorci $^{c}$, \\ Alessandro Pingitore ${ }^{c}$
}

a Clinical and Experimental Department of Medicine and Pharmacology, University of Messina, Messina, Italy http://www.unime.it

b Fondazione G. Monasterio CNR - Regione Toscana, Pisa, Italy, http://www.ftgm.it

c Institute of Clinical Physiology - CNR, Pisa, Italy, http://www.ifc.cnr.it

\section{ARTICLE INFO: \\ RECEIVED: 15 Oct 2015 \\ REVISED: 13 Nov 2015 \\ ACCEPTED: 30 Nov 2015 \\ ONLINE: 23 Dec 2015}

\section{KEYWORDS:}

cardiac magnetic resonance

myocardial infarction

cardiology research

open access

\begin{abstract}
A BSTRACT
Open-access datasets in cardiac magnetic resonance provide the opportunity to approach cardiac research studies in an integrated, multidisciplinary and systematic framework. In this report, we present a CMR dataset obtained by observing patients with previous myocardial infarction.

The results show that scar tissue extent, left ventricular dilatation, and wall motion abnormalities, together with age, are independently associated with cardiac death. Analogous datasets could be used to compare results obtained by imaging techniques identifying patients at higher risk of cardiac death, and to allow generation of a multiparametric score for risk stratification of patients with previous myocardial infarction.
\end{abstract}

\section{Overview}

The assessment of a variety of factors, obtained from clinical examination, bio-humoral analysis, and cardiac imaging allow the prognostic stratification of patients with previous myocardial infarction (MI). ${ }^{1,2}$ In particular, adopting different imaging modalities, left ventricular (LV) systolic dysfunction, LV dilatation and remodelling, the severity and extent of regional wall motion abnormalities, and the extent of necrotic tissue have all been shown to be of prognostic relevance in patients with previous $\mathrm{MI}^{3}$

In particular, the dilatation of both LV end-diastolic volume (LVEDV) and LV end-systolic volume (LVESV) has been related to high incidence of cardiac death, where LVEDV was the only echocardiographic predictor of death in the BEST study. ${ }^{4}$

Cardiac magnetic resonance (CMR) is a non-invasive, non-ionizing, and three-dimensional imaging technique that allows accurate quantifica- 
tion of LV volumes, LV global and regional function, and scar tissue extent in a one-stop-shop modality. ${ }^{5}$

At present, there are no open-access datasets in the CMR field, which provide the opportunity to approach cardiac research studies in an integrated, multidisciplinary, systematic and multicentric framework. Our group has worked for many years in the assessment of cardiac function and morphology by cardiac imaging. An institutional database, IMAGE, has been created to store the most important clinical and instrumental data obtained during hospitalization of patients, affected by cardiovascular disease, from different laboratories (ergometry, echocardiographic, nuclear medicine and hemodynamic). In this report, we present a dataset obtained from CMR in patients with previous MI. Part of these data has been referenced in a previously published study that considered a multiparametric approach for prognostic stratification in patients with previous MI. ${ }^{6}$

\section{Methods}

We studied 231 patients (64.3 \pm 10.9 years old) with previous $\mathrm{MI}$ referred to routine CMR from July 2001 to March 2007. Previous MI was documented by clinical records or by the presence of diagnostic Q-waves in a 12-lead ECG recording, and was older than 3 months in every patient. The inclusion criteria also included angiographically documented coronary stenosis reducing luminal diameter by at least $50 \%$ in one major coronary artery and stable clinical conditions.

In every patient, CMR examination was done 6 months before or after a revascularization procedure. Patients were studied either as out-patient or during the hospitalization period. The clinical characteristics were collected before CMR examination. The study was approved by the local Ethics Committee; the investigation conformed to the principles outlined in the Declaration of Helsinki.

\section{Cardiac Magnetic Resonance Data Acquisition}

CMR was performed using a $1.5 \mathrm{~T}$ whole body scanner (GE Medical Systems, Milwaukee, WI, USA). A 8-channel cardiac phased-array receiver surface coil was used for signal reception. A breath-hold steady-state free-precession ECGtriggered sequence was used to evaluate global LV function. In each patient, a set of contiguous short-axis views were acquired from the mitral plane to the apex and two long-axis views (one vertical and one horizontal) were acquired, with a minimum of 30 cine frames for each slice with the following parameters: slice thickness $8 \mathrm{~mm}$, no gap, eight views per segment, NEX 1 , field of view $40 \mathrm{~cm}$, phase field of view 1 , matrix $224 \times 224$, reconstruction matrix $256 \times 256$, flip angle 458, TR/TE 3.5/1.5, and bandwidth $125 \mathrm{KHz}$. Late gadolinium enhancement (LGE) images were obtained 8-10 min after bolus injection of gadolinium derivates (Omniscanw, Amersham, GE Medical System or Magnevistw, Shering).

Images were acquired in the same short-axis and long-axis slices as used for cine CMR. The fast Gradient Echo Inversion Recovery sequence was utilized with the following parameters: repetition time $4.2 \mathrm{~ms}$, echo time minimum, flip angle 208, matrix $224 \times 224$, number of excitations 1.00 , field of view $36 \mathrm{~mm}$, slice thickness $8 \mathrm{~mm}$, no inter-slice gap. The inversion time was optimized to null signal from the normal myocardium.

\section{Cardiac Magnetic Resonance Data Analysis}

To determine LV function, endocardial borders were manually drawn on all LV short-axis images by means of previously validated software (Massw, MEDIS, The Netherlands). The LV mass, LVESV, and LVEDV, normalized for the body mass index $(\mathrm{mL} / \mathrm{m} 2)$, were then calculated and the LVEF was derived. LV was divided into 17 segments including six basal, six middle, four distal segments, and the apex. ${ }^{7}$ Wall motion of each segment was graded semi-quantitatively according to a fourpoint scale where 1 is normal, 2 is hypokinetic, 3 is akinetic, and 4 is dyskinetic. The score of the segments belonging to an LV wall was averaged to derive the wall motion score index (WMSI). The ratio mass/EDV was considered as an LV remodelling index. ${ }^{8,9}$ The global LGE extent was measured using a semiautomatic, software. ${ }^{10}$ In each image, boundaries of contrast-enhanced areas were automatically traced (using a signal intensity cut-off of $>5$ SD over the average of normal remote myocardium) and manually corrected when needed. The reproducibility of this method has been previously validated. ${ }^{10}$ The transmural LGE extent was measured by standard techniques. ${ }^{11}$ For each segment, the LGE transmural extent was expressed as percentage of total segment area and clustered as follows: $1-25 ; 26-50 ; 51-75$; or $>75 \%$. Furthermore, LVEDV, WMSI, and LGE extent were then dichotomized according to their median values.

\section{Follow-up Data Acquisition}

A questionnaire compiled by a clinical physician during periodic ambulatory work up in our institute or telephone contact was used for follow-up 
acquisition of data. The length of follow-up was from 3 months to $\sim 8$ years. The events considered were cardiac death, and appropriate implantable cardiac defibrillator (ICD) shock. The cause of death was derived from medical records or death certificates. The definition of cardiac death required the documentation of significant arrhythmia or cardiac rest or death attributable to congestive heart failure or $\mathrm{MI}$ in the absence of any other precipitating factor. A complete interrogation of the ICD was performed by the referring physician in order to confirm the appropriateness of the shock. ${ }^{12}$

\section{Data Records}

The data in the accompanying spreadsheet is organised in the following columns:

A: It shows the ID of the subject.

B: It shows the sex of the subjects ( $1=$ male; $0=$ female).

C: It shows the age of the subjects at the time of CMR.

D: It shows the presence (1) or not (0) of diabetes.

E: It shows the presence (1) or not (0) of hypercholesterolemia.

F: It shows the presence (1) or not (0) of hypertension.

G: It shows the presence (1) or not (0) of smoking habits.

H: It shows body weight of patients in kilograms.

I: It shows height of patients in centimetres.

J: It shows Body Mass Index (BMI) as value derived from the weight and height of an individual.

K: It shows the time, expressed in years, between $\mathrm{MI}$ and $\mathrm{CMR}$.

L: It shows the left ventricular ejection fraction, in $\%$, that was measured by CMR.

M: It shows the end-diastolic volume, expressed in $\mathrm{ml}$, measured by CMR.

$\mathbf{N}$ : It shows the end-systolic volume, expressed in $\mathrm{ml}$, measured by CMR.

O: It shows the Wall Motion Score Index (WMSI).

P: It shows the Late Gadolinium Enhancement (LGE) transmural extent $<50 \%$ (number of segments) evaluated by CMR.
Q: It shows the LGE transmural extent $>50 \%$ (number of segments) evaluated by CMR.

R: It shows the LGE total segment $(<50 \%$ and $>50 \%$ ) evaluated by CMR.

S: It shows the dates of censoring for end of the follow-up.

T: It shows the presence of cardiac death (or appropriate implantable cardiac defibrillator, ICD therapy) (1) or not (0).

$\mathrm{U}$ : It shows the presence of non-cardiac death (1) or not (0).

V: It shows the date of death.

W: It shows the time expressed in years from CMR to Follow Up (FU) or death.

\section{Validation}

Using the CMR dataset described in this paper, we showed that a multiparametric CMR approach, including the measure of scar tissue extent, LV enddiastolic volume and regional wall motion abnormalities, together with age, fine-tunes risk stratification of patients with previous MI. In particular, when the WMSI is 1.7 , the extent of scar tissue exceeds $12.7 \%$ of the LV mass, and LV volume is $.105 \mathrm{~mL} / \mathrm{m} 2$, the survival free of cardiac death is lower than when two, one, or none of these factors are present. ${ }^{6}$

\section{Use and Potential Reuse}

The dataset described in this paper, or part of it, has been already used to assess the CMR role in patients with previous $\mathrm{MI}$, specifically regarding the relationship between electrocardiographic signs of previous MI and LGE global extent and location, and prognosis. Recently, in patients with inferior and/or lateral $\mathrm{MI}$, a prominent $\mathrm{T}$ wave in V2 with respect to V6 corresponded to a greater infarct extent in the lateral wall. ${ }^{13}$ Further, the potential to assess transmural extent of MI with LGE technique allowed us to demonstrate that necrotic and viable myocardium coexistence within the same wall segments predicted occurrence of non-sustained ventricular tachycardia in patients with previous $\mathrm{MI}$ and without left ventricular dilatation. $^{14}$

CMR is considered the non-invasive and nonionizing gold standard imaging technique to assess both right and left ventricles. With this dataset, we showed that right ventricular dysfunction is an independent and incremental predictor of cardiac events in patients with previous MI. In these patients, right ventricular dysfunction does not nec- 
essarily mean right ventricular infarction scar, but likely reflects the effects of hemodynamic and biohumoral factors activated in patients with left ventricular dysfunction. ${ }^{15}$

\section{Conclusion}

In conclusion, the CMR and clinical dataset of patients with previous $\mathrm{Ml}$, attached in this paper, can be reused to assess the CMR role in the prognostic stratification of patients with previous $\mathrm{MI}$ in a large and multicentre study population. In addition, data sharing of clinical, bio-humoral, and imaging variables could allow generation of a multiparametric score to identify patients with higher risk of major cardiac events and to define targeted therapeutic interventions as implanted cardioverter defibrillator.

\section{Data Files}

The data described in this paper is presented in an openly accessible spreadsheet: "01305_Cardiac_ Magnetic_Resonance_Imaging.xIs".

The file is available also in the Open Document Spreadsheet format.

\section{Acknowledgements}

The preparation of this manuscript was supported by the EU-funded project OpenScienceLink (Grant agreement \# 318652).

\section{References}

${ }^{1}$ Huikuri HV, Mäkikallio TH, Raatikainen MJ, Perkiömäki J, Castellanos A, Myerburg RJ. Prediction of sudden cardiac death: appraisal of the studies and methods assessing the risk of sudden arrhythmic death. Circulation 2003;108(1): 110-5. PMID: 12847054 doi: 10.1161/01.CIR. 0000077519.18416 .43 .

${ }^{2}$ Rahimtoola SH, Dilsizian V, Kramer CM, Marwick TH, Vanoverschelde JL. Chronic ischemic left ventricular dysfunction: from pathophysiology to imaging and its integration into clinical practice. JACC Cardiovasc Imaging 2008;1(4):536-55. doi: 10.1016/j.jcmg.2008.05.009.

${ }^{3}$ Reynolds MR, Josephson ME. MADIT II (second multicenter automated defibrillator implantation trial) debate: risk stratification, costs, and public policy. Circulation 2003;108(15):1779-83. doi: 10.1161/01.CIR.0000086777.82110.F5.

${ }^{4}$ Grayburn PA, Appleton CP, DeMaria AN, Greenberg B, Lowes B, Oh J, Plehn JF, Rahko P, St John Sutton $M$, Eichhorn EJ. Echocardiographic predictors of morbidity and mortality in patients with advanced heart failure: the beta-blockers evaluation of survival trial (BEST). J Am Coll Cardiol. 2005;45(7):1064-71. doi: 10.1016/j.jacc. 2004.12.069.

${ }^{5}$ Pennell DJ, Sechtem UP, Higgins CB, Manning WJ, Pohost GM, Rademakers FE, Van Rossum AC, Shaw $L$, Yucel EK. Clinical indications for cardiovascular magnetic resonance (CMR): consensus panel report. Eur Heart J. 2004;25(21):1940-65. doi: 10.1016/j.ehj.2004. 06.040.

${ }^{6}$ Di Bella G, Siciliano $V$, Aquaro GD, Molinaro $S$, Lombardi $M$, Carerj S, Landi $P$, Rovai D, Pingitore A. Scar extent, left ventricular end-diastolic volume, and wall motion abnormalities identify highrisk patients with previous myocardial infarction: a multiparametric approach for prognostic stratification. Eur Heart J. 2013;34(2):104-11. doi: 10.1093/eurheartj/ ehs037.

7 Cerqueira MD, Weissman NJ, Dilsizian V, Jacobs AK, Kaul S, Laskey WK, Pennell D J, Rumberger JA, Ryan T, Verani MS, American Heart Association Writing Group on Myocardial Segmentation and Registration for Cardiac Imaging. Standardized myocardial segmentation and nomenclature for tomographic imaging of the heart: a statement for healthcare professionals from the Cardiac Imaging Committee of the Council on Clinical Cardiology of the American Heart Association. Circulation 2002; 105(4):539-42. doi: 10.1161/hc0402.102975.

${ }^{8}$ Hees PS, Fleg JL, Lakatta EG, Shapiro EP. Left ventricular remodeling with age in normal men versus women: novel insights using three-dimensional magnetic resonance imaging. Am J Cardiol. 2002;90(11):1231-36. doi: 10.1016/S0002-9149 (02)02840-0.

${ }^{9}$ Watzinger N, Lund GK, Higgins CB, Wendland MF, Weinmann HJ, Saeed M. The potential of contrast-enhanced magnetic resonance imaging for predicting left ventricular remodeling. J Magn Reson Imaging 2002;1686):633-40. doi: 10.1002/ jmri.10206.

${ }^{10}$ Positano V, Pingitore A, Giorgetti A, Favilli B, Santarelli MF, Landini L, Marzullo P, Lombardi M. A fast and effective method to assess myocardial necrosis by means of contrast magnetic resonance imaging. J Cardiovasc Magn Reson. 2005;7(2):48794. PMID: 15881533.

11 Kim RJ, Wu E, Rafael A, Chen EL, Parker MA, Simonetti O, Klocke FJ, Bonow RO, Judd RM. The use of contrast-enhanced magnetic resonance imaging to identify reversible myocardial dysfunction. N Engl J Med. 2000;343(20):1445-53. doi: 10.1056/NEJM200011163432003. 
12 Aquaro GD, Pingitore A, Strata E, Di Bella G, Molinaro S, Lombardi M. Cardiac magnetic resonance predicts outcome in patients with premature ventricular complexes of left bundle branch block morphology. J Am Coll Cardiol. 2010;56(15): 1235-43. doi: 10.1016/j.jacc.2010.03.087.

${ }^{13}$ Rovai D, Rossi G, Pederzoli L, Aquaro GD, Di Bella G, Pingitore A. Prominent T wave in V2 with respect to $V 6$ as a sign of lateral myocardial infarction. Int J Cardiol. 2015;189:148-52. doi: 10.1016/j.ijcard.2015.04.050.

${ }^{14}$ Di Bella G, Passino C, Aquaro GD, Rovai D, Strata E, Arrigo F, Emdin M, Lombardi M, Pingitore
A. Different substrates of non-sustained ventricular tachycardia in post-infarction patients with and without left ventricular dilatation. J Card Fail. 2010;16(1):61-8. doi: 10.1016/j.cardfail.2009.09. 001.

${ }^{15}$ Di Bella G, Siciliano V, Aquaro GD, De Marchi D, Rovai D, Carerj S, Molinaro S, Lombardi M, Pingitore A. Right ventricular dysfunction: an independent and incremental predictor of cardiac deaths late after acute myocardial infarction. Int J Cardiovasc Imaging. 2015;31(2):379-87. doi: 10.1007/ s10554-014-0559-9. 